\title{
Dense molecular clouds in the SN 2008fp host galaxy
}

\author{
N. L. J. $\operatorname{Cox}^{1}$ and F. Patat ${ }^{2}$ \\ 1 Instituut voor Sterrenkunde, KU Leuven, Celestijnenlaan 200 D, 3001 Leuven, Belgium \\ e-mail: nick.cox@ster.kuleuven.be \\ 2 European Organization for Astronomical Research in the Southern Hemisphere (ESO), Karl Schwarzschild-Str. 2, \\ 85748 Garching bei München, Germany \\ e-mail: fpatat@eso.org
}

Received 1 March 2012 / Accepted 17 March 2014

\begin{abstract}
Context. Supernovae (SNe) offer a unique opportunity to study physical properties, small-scale structure, and complex organic chemistry of the interstellar medium (ISM) in different galaxies.

Aims. Interstellar absorption features, such as atomic and molecular lines as well as diffuse interstellar bands (DIBs), can be used to study the physical properties of extra-galactic diffuse interstellar clouds.

Methods. We used optical high-resolution spectroscopy to study the properties of the ISM in the SN2008fp host galaxy, ESO 428G14. The properties of intervening dust were investigated via spectropolarimetry.

Results. The spectra of SN 2008fp reveal a complex of diffuse atomic clouds at radial velocities in line with the systematic velocities of the host galaxy. In addition, a translucent $\left(A_{\mathrm{V}} \sim 1.5 \mathrm{mag}\right.$ ) cloud is detected at a heliocentric velocity of $1770 \mathrm{~km} \mathrm{~s}^{-1}$ (redshifted by $70 \mathrm{~km} \mathrm{~s}^{-1}$ with respect to the system velocity). This cold dense cloud is rich in dense atomic gas tracers, molecules, as well as DIBs. We have detected both $\mathrm{C}_{2}$ and $\mathrm{C}_{3}$ for the first time in a galaxy beyond the Local Group. The $\mathrm{CN}(0,0)$ band-line ratios are consistent with an excitation temperature of $\mathrm{T}=2.9 \pm 0.4 \mathrm{~K}$. The interstellar polarisation law deviates significantly from what is observed in the Galaxy, indicating substantial differences in the host dust/size composition. No variations over a period of about one month are observed in any of the ISM tracers.

Conclusions. The lack of variability in the extra-galactic absorption line profiles implies that the absorbing material is not circumstellar and thus not directly affected by the $\mathrm{SN}$ event. It also shows that there are no significant density variation in the small-scale structure of the molecular cloud down to $100 \mathrm{AU} . \mathrm{C}_{2}$ is used to probe the cold diffuse ISM density and temperature. Here we also use observations of $\mathrm{CN}$ in a distant galaxies, though for now still in a limited way, for in situ measurements of the cosmic background radiation temperature.
\end{abstract}

Key words. supernovae: individual: SN 2008fp - ISM: lines and bands - ISM: molecules

\section{Introduction}

Multi-epoch high-resolution optical spectroscopy of nearby Type Ia supernovae have been presented in relation to the study of $\mathrm{NaI}$ and $\mathrm{Ca}$ II to probe the circumstellar matter associated with the progenitors of these events. This has led to the discovery of time variability in high-resolution $\mathrm{Na}$ I absorption line systems for some supernovae (see e.g. Patat et al. 2007; Simon et al. 2009). Several similar events were detected using lowresolution spectroscopy (Blondin et al. 2009; Stritzinger et al. 2010). However, not all Type Ia SNe show this behaviour (e.g. Patat et al. 2013). Furthermore, Sternberg et al. (2011) found that for a large sample of Type Ia SNe, the absorbing material (Na I) tends to be blue-shifted with respect to the strongest sodium absorption line, which has been suggested as an indication of outflows from the supernova progenitor systems. This is expected for single-degenerate (SD) systems but is much less probable for double-degenerate (DD) systems. This result needs to be taken with caution as the strongest sodium absorption component does not necessarily have the same velocity as the disk gas at the position of the supernova (e.g. Ritchey \& Wallerstein 2012).

$\star$ This paper is based on observations made with ESO Telescopes at Paranal Observatory under program IDs 081.D-0558, 081.D-0697, and 082.D-0004.
Phillips et al. (2013) showed that some supernovae - all of which were classified as blue-shifted - display anomalously high Na I column densities with respect to the amount of dust extinction, suggesting a relation to the outflowing circumstellar gas. Pathways for producing this enhancement were given for both the SD and DD scenarios.

The increasing availability of high-resolution highsensitivity spectra (in particular when averaging over multiple epochs) has provided the possibility to study the physical properties of the interstellar medium (ISM) in other galaxies in detail. Until recently, only a few supernovae, such as SN 1987A, could be studied in detail. Now, bright stars in the Magellanic Clouds (Cox et al. 2006; Welty et al. 2006) and the Andromeda and Triangulum galaxies (Cordiner et al. 2008a,b) are accessible - with some effort - to optical (high-resolution) spectroscopy. To proceed in studying distant galaxies it is becoming readily possible to use nearby $\mathrm{SNe}$, similar to the exploitation of SN 1987A to study the LMC (Grewing et al. 1987; Pettini 1988), as background candles to probe the (physical) properties of the ISM such as dust extinction and UV field strength in their host galaxy through the observation of atoms, molecules, and diffuse interstellar bands (DIBs). However, sufficiently bright $\mathrm{SNe}$ are required to obtain high-S/N spectra at high-resolution, which limits the number of possibilities for 
such studies. Furthermore, not all SNe will probe significant columns of interstellar matter, in particular if they are located in the approaching side of the respective galaxy.

Although rare, an increasing number of SNe have been observed with intermediate to high spectral resolution to probe the atomic and molecular diffuse ISM, including DIBs, in the disks and halos of galaxies beyond the Local Group, such as the Centaurus group (D'Odorico et al. 1985, 1989; Rich 1987), the Virgo cluster (Steidel et al. 1990; Meyer \& Roth 1991) and the M81 group (Bowen et al. 1994). The most detailed studies of both resolved molecular absorption lines and resolved DIB profiles arising from the cold diffuse ISM in galaxies beyond the Local Group were presented in Sollerman et al. (2005) and Cox $\&$ Patat (2008). In addition, interstellar absorption features such as the UV bump and the strong 5780 DIB have also been detected in distant damped Ly $\alpha$ (DLA) systems (Junkkarinen et al. 2004; York et al. 2006; Ellison et al. 2008; Srianand et al. 2013).

Here we present a detailed study of the properties of the ISM in the SN host galaxy and the Galactic halo as probed by SN 2008fp. The spectra were obtained as part of an ongoing campaign to observe atomic line variablity in bright Type Ia $\mathrm{SNe}$ in an effort to distinguish between the SD and DD scenarios for Type Ia SNe (cf. Patat et al. 2013). In Sect. 2 we present the observations and data reduction and give basic properties of the $\mathrm{SN}$ and its host galaxy. The detected Galactic and extra-galactic interstellar absorption lines are presented in Sect. 3. In Sect. 4 we discuss the molecular content, physical cloud conditions, interstellar line variability and the presence and behaviour of extragalactic molecules and DIBs in the SN host galaxy. We conclude with a summary in Sect. 5 .

\section{Observations}

High-resolution UVES spectra were obtained in service mode for supernova 2008fp. SN 2008fp is a normal Type Ia SN (Wang et al. 2008; Stritzinger et al. 2011) discovered by Pignata et al. (2008) in the galaxy ESO 428-G14, which is at $\sim 26 \mathrm{Mpc}$. The reported galaxy type is $\operatorname{SABr}(0)$-pec (De Vaucouleurs et al. 1991) and Seifert 2. Its heliocentric system velocity is $1698 \pm 20 \mathrm{~km} \mathrm{~s}^{-1}$ (Wegner et al. 2003). SN 2008fp is located in the sky at a Galactic longitude of $241.9^{\circ}$ and at a Galactic latitude of $-8.0^{\circ}$. Phillips et al. (2013) derived $A_{\mathrm{V}}=0.71_{-0.08}^{+0.10} \mathrm{mag}$ and $R_{\mathrm{V}}=1.20_{-0.14}^{+0.26}$ from a light-curve analysis.

Spectra were obtained at different phases of the SN evolution. SN 2008fp was observed at 6, 11, 17, and 39 days after the reported discovery. These correspond to days $-2,+3,+9$, and +31 with respect to the optical maximum light attained on MJD $\approx 54728$ (Stritzinger et al. 2011). Thus, the set of spectra covers approximately one month of temporal evolution. Table 1 gives a summary of the observations. The data were processed with the standard UVES pipeline routines. All individual spectra were corrected for the heliocentric velocity before averaging of individual frames at each epoch. In addition, we noticed a small shift (of about 1 pixel) in peak velocities of the individual cloud components between spectra taken at different epochs. Further analysis of the sky emission lines (at 5889.95, 5895.924, and $6304 \AA$ ) as well as the Galactic foreground absorption lines showed a small deviation in wavelength calibration between exposures taken with the red arm. The spectra taken with the blue arm did not suffer from this small shift. All individual spectra were corrected for this effect. The sky emission lines and telluric absorption lines were measured to confirm the obtained spectral resolution of $6 \mathrm{~km} \mathrm{~s}^{-1}$. The achieved signal-to-noise ratio $(\mathrm{S} / \mathrm{N})$
Table 1. Observation log for SN 2008fp.

\begin{tabular}{lllll}
\hline \hline Target & $\begin{array}{l}\text { Date } \\
\text { (yyyy-mm-dd) }\end{array}$ & $\begin{array}{l}\text { Epoch } \\
\text { (day) }\end{array}$ & UVES setting $^{a}$ & $\begin{array}{l}\text { Exposure } \\
\text { time (s) }\end{array}$ \\
\hline SN 2008fp & $2008-09-17$ & +6 & Blue & 7200 \\
& & & $\begin{array}{l}\text { Red1 } \\
\text { Red2 }\end{array}$ & 3600 \\
& $2008-09-22$ & +11 & Blue & 3600 \\
& & & Red1 & 7200 \\
& & & Red2 & 3600 \\
& $2008-09-28$ & +17 & Blue, Red1 & 3600 \\
& $2008-10-20$ & +39 & Blue, Red1 & 7200 \\
\hline
\end{tabular}

Notes. ${ }^{(a)}$ UVES spectral coverage (approximately) for each setting: blue [3300-4550 ̊], red1 [4600-5600 ̊ + 5700-6650 ̊], red2 [6700$8500 \AA+8650-10500 \AA]$. The observed epoch is calculated as the number of days since 2008-09-11, the date of discovery.

Table 2. Galactic $\mathrm{CH}^{+}$and diffuse bands in the lines-of-sight towards SN 2008fp.

\begin{tabular}{lll}
\hline \hline Line & $E W(\mathrm{m \AA})^{a}$ & $v\left(\mathrm{~km} \mathrm{~s}^{-1}\right)$ \\
\hline $\mathrm{CH}^{+}$ & $5 \pm 2$ & 67.4 \\
$5780.55 \mathrm{DIB}$ & $115 \pm 10$ & 59.6 \\
$5797.08 \mathrm{DIB}$ & $25 \pm 3$ & 68.3 \\
$5849.81 \mathrm{DIB}$ & $19 \pm 2$ & 50.7 \\
$6613.63 \mathrm{DIB}$ & $38 \pm 7$ & 62.1 \\
\hline
\end{tabular}

Notes. ${ }^{(a)}$ Equivalent width uncertainties have been computed following the prescription in Vos et al. (2011).

in the averaged spectra is about 60 to 80 for the blue range (e.g. for $\mathrm{CN}, \mathrm{CaI}, \mathrm{CH}, \mathrm{CH}^{+}$), while for the red range it is about 90 to 120 (e.g. 5797,5780 and $6613 \AA$ DIBs). In the near-infrared it is again lower; the $\mathrm{S} / \mathrm{N}$ is about $50-60$ for the $\mathrm{C}_{2}$ transitions.

\section{Interstellar medium towards SN 2008fp}

First inspection of the UVES spectra of SN 2008fp revealed immediately that the line-of-sight probes a rich host-galaxy ISM. Atoms, molecules, and DIBs are detected at radial velocities corresponding to that of the Milky Way and the host galaxy, ESO 428-G14. Next we present the observed interstellar lines in the Milky Way and SN host galaxy. The Galactic and extragalactic Ca II and $\mathrm{Na}$ I lines are discussed in more depth in Cox et al. (in prep.), which will present an extensive analysis of both Galactic and extra-galactic sodium and calcium doublets for a larger sample of SNe.

\subsection{Galactic foreground ISM}

In addition to the strong Galactic $\mathrm{Ca}$ II and $\mathrm{Na}$ I components present between 0 and $120 \mathrm{~km} \mathrm{~s}^{-1}$, we also detected $\mathrm{CH}^{+}$ and several DIBs. Equivalent widths and radial velocities are given in Table 2. Typical radial velocities are between 50 and $70 \mathrm{~km} \mathrm{~s}^{-1}$. Using the DIB strength versus interstellar reddening relation for field stars derived by Luna et al. (2008), the 5797 and $6613 \AA$ DIB strengths give a Galactic foreground reddening of $\sim 0.15-0.20 \mathrm{mag}$. This is in excellent agreement with the Galactic foreground reddening, $E_{(B-V)}=0.2 \mathrm{mag}$, estimated from the all-sky dust extinction map (Schlegel et al. 1998). The total column densities for Galactic Ca II is $1.4 \times 10^{13} \mathrm{~cm}^{-2}$. 
Interstellar lines toward SN2008fp

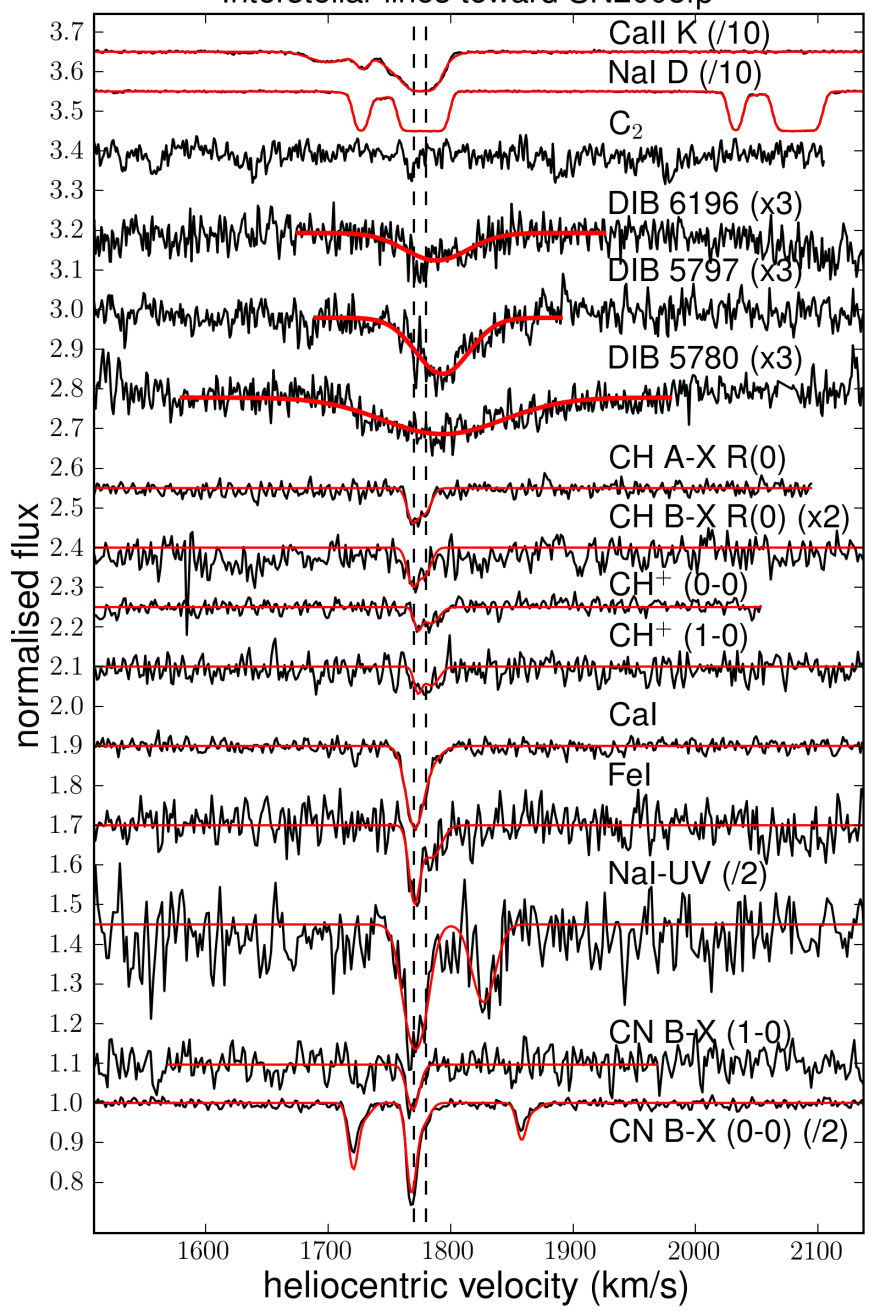

Fig. 1. Interstellar absorption lines towards SN 2008fp. Synthetic line profiles obtained with VPFIT are shown as solid red curves overplotted on the observed spectra. Details of the models are given in Table 3. The vertical dashed line are drawn at 1770 and $1778 \mathrm{~km} \mathrm{~s}^{-1}$, the average peak radial velocity for the strongest interstellar components. The 5797 DIB and $\mathrm{CH}^{+}$appear slightly red-shifted with respect to the average radial velocity. The shift for 5797 DIB corresponds to roughly $0.4 \AA$ or $20 \mathrm{~km} \mathrm{~s}^{-1}$. Two velocity components separated by $\sim 8 \mathrm{~km} \mathrm{~s}^{-1}$ are discerned in the absorption profiles of $\mathrm{CN}, \mathrm{CH}$, and $\mathrm{CH}^{+}$.

\subsection{Supernova host galaxy ISM}

Both Na ID and Ca II HK are detected at radial velocities corresponding to those of the host galaxy (Fig. 1). The main absorption complex is between +50 to $+100 \mathrm{~km} \mathrm{~s}^{-1}$ with respect to the system velocity at $1698 \mathrm{~km} \mathrm{~s}^{-1}$. In addition, many other atomic and molecular species are detected at velocities corresponding to those of the main components of $\mathrm{Na}$ I, as shown clearly in Fig. 1. We used VPFIT ${ }^{1}$ to obtain densities for the atomic and molecular species. Whenever possible, multiple transitions of a particular species were used to correct for line saturation and improve the constraint of the fit. The results for the central radial velocities and the column density or equivalent width for the atomic and molecular species and DIBs, respectively, are listed in Table 3. A detailed discussion of each of the atomic species, molecules, and

\footnotetext{
1 Carswell \& Webb; http://www.ast.cam.ac.uk/ rfc/vpfit. html
}

Table 3. Atomic, molecular, and diffuse bands detected in the line-ofsight toward SN 2008fp.

\begin{tabular}{|c|c|c|c|}
\hline Line & $\begin{array}{l}v_{\text {helio }}{ }^{a} \\
\left(\mathrm{~km} \mathrm{~s}^{-1}\right)\end{array}$ & $\begin{array}{c}E W \\
(\mathrm{~m} \AA) \\
\end{array}$ & $\begin{array}{l}\log N^{a} \\
\left(\mathrm{~cm}^{-2}\right)\end{array}$ \\
\hline Na I UV (doublet) & $1771.5 \pm 0.7$ & $\begin{array}{r}128 \pm 26 \\
67 \pm 13\end{array}$ & $14.43 \pm 0.03$ \\
\hline Ca II HK (doublet) & $1700-1780$ & & $13.39 \pm 0.02$ \\
\hline Fe I (3719.9Å) & $1770.4,1784.4$ & $44 \pm 5$ & $12.96 \pm 0.03$ \\
\hline Fe I $(3859.9 \AA)$ & & $26 \pm 6$ & \\
\hline Ca I & $1770.5,1780.7$ & $60 \pm 5$ & $11.35 \pm 0.02$ \\
\hline Na I D (doublet) & $1726-1790$ & & $14.35 \pm 0.06$ \\
\hline K I (doublet) & $1770-1791$ & & $12.03 \pm 0.03$ \\
\hline Ti II & 1775 & $46 \pm 9$ & $12.1 \pm 0.1$ \\
\hline CN B-X $(0-0) R_{0}$ & 1767.8 & $84 \pm 3$ & $13.40 \pm 0.03$ \\
\hline CN B-X $(0-0) R_{1}$ & 1768.7 & $37 \pm 3$ & $13.12 \pm 0.06$ \\
\hline CN B-X $(0-0) P_{1}$ & 1768.3 & $19 \pm 2$ & \\
\hline CN B-X $(1-0) R_{0}$ & 1769.3 & $18 \pm 4$ & $13.7 \pm 0.1$ \\
\hline $\mathrm{CH}^{+} \mathrm{A}-\mathrm{X}(0-0) \mathrm{R}(0)$ & $1773.2,1784.3$ & $16 \pm 3$ & $13.23 \pm 0.22$ \\
\hline $\mathrm{CH}^{+} \mathrm{A}-\mathrm{X}(1-0) \mathrm{R}(0)$ & & $8 \pm 2$ & \\
\hline CH A-X (0-0) & $1768.9,1779.2$ & $27 \pm 4$ & $13.45 \pm 0.36$ \\
\hline CH B-X (0-0) & & $11 \pm 3$ & \\
\hline $\mathrm{C}_{2} \mathrm{~A}-\mathrm{X}(2-0) \mathrm{R} 0$ & 1766.8 & $16 \pm 2$ & $13.23 \pm 0.06^{b}$ \\
\hline $\mathrm{C}_{2} \mathrm{~A}-\mathrm{X}(2-0) \mathrm{R} 2$ & 1768.9 & $19 \pm 2$ & $13.70 \pm 0.05^{b}$ \\
\hline $\mathrm{C}_{2} \mathrm{~A}-\mathrm{X}(2-0) \mathrm{R} 4-\mathrm{R} 8$ & 1768.1 & $15 \pm 2$ & $13.68 \pm 0.06^{b}$ \\
\hline $\mathrm{C}_{2} \mathrm{~A}-\mathrm{X}(2-0) \mathrm{R} 6$ & 1769.5 & $8 \pm 3$ & $13.44 \pm 0.20^{b}$ \\
\hline $\mathrm{C}_{2} \mathrm{~A}-\mathrm{X}(2-0) \mathrm{Q} 2$ & 1765.9 & $25 \pm 3$ & $13.72 \pm 0.06^{b}$ \\
\hline $\mathrm{C}_{2} \mathrm{~A}-\mathrm{X}(2-0) \mathrm{Q} 4$ & 1770.2 & $21 \pm 3$ & $13.64 \pm 0.04^{b}$ \\
\hline $\mathrm{C}_{2} \mathrm{~A}-\mathrm{X}(2-0) \mathrm{Q} 6$ & 1772.6 & $15 \pm 3$ & $\begin{array}{l}13.50 \pm 0.10^{b} \\
\Sigma(N)= \\
14.29 \pm 0.07^{b}\end{array}$ \\
\hline $\mathrm{C}_{3} \tilde{\mathrm{A}}-\tilde{\mathrm{X}}(Q$-branch $)$ & 1768.0 & $20 \pm 5$ & $13.23 \pm 0.15^{c}$ \\
\hline $5780.55 \mathrm{DIB}$ & $1787 \pm 10$ & $81 \pm 5$ & \\
\hline $5797.08 \mathrm{DIB}$ & $1780 \pm 5$ & $60 \pm 10$ & \\
\hline 5849.81 DIB & $1778 \pm 5$ & $34 \pm 5$ & \\
\hline 6196.00 DIB & $1776 \pm 14$ & $29 \pm 6$ & \\
\hline 6283.85 DIB & $1796 \pm 15$ & $180 \pm 30$ & \\
\hline
\end{tabular}

Notes. ${ }^{(a)}$ Column densities and radial velocities for the atomic species $\mathrm{CH}, \mathrm{CH}^{+}$, and $\mathrm{CN}$ are obtained from multiple-component profile fitting using VPFIT. Whenever possible, multiple transitions (e.g. doublets) of a single species were fitted simultaneously. For Fe I, $\mathrm{CH}^{+}$, and $\mathrm{CH}$, two velocity components (see table) were required to achieve the best fit. ${ }^{(b)}$ Details for $N\left(\mathrm{C}_{2}\right)$ in Sect. 3.2.2. ${ }^{(c)} N\left(\mathrm{C}_{3}\right)$ is derived following the procedure of Oka et al. (2003) (Sect. 3.2.2).

DIBs in the SN 2008fp host galaxy are given in the subsequent subsections.

\subsubsection{Extra-galactic atomic species}

The strongest absorption features near the systemic velocity of the host galaxy are from $\mathrm{Na}$ I and Ca II. These are shown in the top two traces of Fig. 1. For both species the transitions between 1750 and $1800 \mathrm{~km} \mathrm{~s}^{-1}$ are saturated. Two weaker components are present at 1700 and $1730 \mathrm{~km} \mathrm{~s}^{-1}$. In addition to these strong absorption systems the weaker transitions of Ca I, K I, and $\mathrm{Na}$ I UV are also detected around $1770-1780 \mathrm{~km} \mathrm{~s}^{-1}$ (Fig. 1 and Table 3). Ti II is only tentatively detected at a radial velocity of $1775 \mathrm{~km} \mathrm{~s}^{-1}$ with an equivalent width of $46 \pm 9 \mathrm{~m} \AA$. Other interstellar atomic species, such as Li I and KIUV, are not detected. Total column densities derived with VPFIT are listed in Table 3. Whenever possible, multiple transitions, such as those for doublets, were included in the VPFIT fitting procedure to 
A\&A 565, A61 (2014)

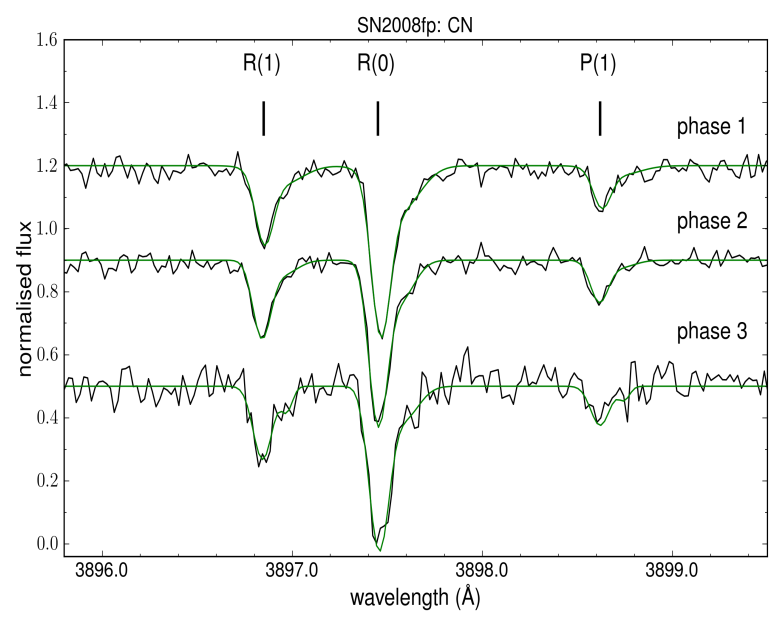

Fig. 2. CN R-X $(0,0)$ spectrum observed toward SN 2008fp at first 3 epochs. The indicated line transitions are plotted at a common radial velocity of $1770 \mathrm{~km} \mathrm{~s}^{-1}$, corresponding to the velocity of atomic and di-atomic lines associated to the host galaxy. The VPFIT model is overplotted as a solid green curve.

take saturation into account and thus improve the robustness of the total column densities. The uncertainties in equivalent width were derived from the continuum $\mathrm{S} / \mathrm{N}$, while uncertainties on the column densities - except for $\mathrm{C}_{2}$ and $\mathrm{C}_{3}$ - are those reported by VPFIT. This results in differences in relative uncertainties for equivalent widths and column densities.

\subsubsection{Extra-galactic molecular lines and bands}

$\mathrm{CH}$. The strong $\mathrm{CH}$ A-X $(0-0)$ and $\mathrm{B}-\mathrm{X}(0-0)$ absorption lines at $4300.313 \AA$ and $3886.4132 \AA$, respectivley, are clearly detected towards SN 2008fp (Fig. 1). Two velocity components separated by $\sim 8 \mathrm{~km} \mathrm{~s}^{-1}$ are present. To take into account mild saturation of the strongest line, both lines are fitted simultaneously with VPFIT, which gives $N(\mathrm{CH})=2.8_{-1.6}^{+3.7} \times 10^{13} \mathrm{~cm}^{-2}$.

$\mathrm{CH}^{+}$. Both the $\mathrm{CH}^{+} \mathrm{A}-\mathrm{X}(0-0) \mathrm{R}(0)$ and A-X (1-0) $\mathrm{R}(0)$ transitions at 4232.548 and $3957.70 \AA$, respectively, are detected (Fig. 1). Two velocity components separated by $\sim 8 \mathrm{~km} \mathrm{~s}^{-1}$ are present. A simultaneous fit to the two transitions gives $N\left(\mathrm{CH}^{+}\right)=1.7_{-0.7}^{+1.1} \times 10^{13} \mathrm{~cm}^{-2}$.

CN. The $R_{0}, R_{1}$, and $P_{1}$ lines of the $\mathrm{CN}(0,0) B^{2} \Sigma^{+}-X^{2} \Sigma^{+}(0,0)$ vibrational band, as well as the $R_{0}$ line of the CN B-X (1-0) band at $3579.963 \AA$, are detected towards SN 2008fp (Figs. 1 and 2, and Table 4). The $R_{0}$ and $R_{1}$ line profiles have a weak red wing, suggesting that the absorption system consists of at least two velocity components separated by $\sim 9 \mathrm{~km} \mathrm{~s}^{-1}$ (similar to the velocity structure seen in $\mathrm{CH}$ and $\mathrm{CH}^{+}$line profiles). The $R_{0}$ profile peaks at a radial velocity of $\sim 1768 \mathrm{~km} \mathrm{~s}^{-1}$.

From the VPFIT line fitting we derive a total column density $N(\mathrm{CN} B-\mathrm{X}(0-0))=2.6_{-0.6}^{+0.9} \times 10^{13} \mathrm{~cm}^{-2}$. The Doppler width is $b=3.7 \pm 1.6 \mathrm{~km} \mathrm{~s}^{-1}$ for the main component. The second weaker component appears broader, but is poorly constrained. This is similar to the curve-of-growth-corrected column density, $N(\mathrm{CN}$ $\mathrm{B}-\mathrm{X}(0,0)) \approx 2.5 \times 10^{13} \mathrm{~cm}^{-2}$ (Sect. 4.6). We find a higher column density for the weaker line, CN B-X $(1-0)$ line of $N(\mathrm{CN})=$ $(5.0 \pm 1.1) \times 10^{13} \mathrm{~cm}^{-2}$ (or $\log N(\mathrm{CN})=13.7$; optical thin limit,
Table 4. Measured equivalent widths and curve-of-growth corrected column densities for $\mathrm{CN}(0,0)$ band at each epoch.

\begin{tabular}{|c|c|c|c|c|c|c|c|}
\hline \multirow{2}{*}{$\begin{array}{l}\text { Epoch } \\
\text { (days) }\end{array}$} & & \multicolumn{3}{|c|}{$W(\mathrm{~m} \AA)$} & \multirow{2}{*}{$\begin{array}{l}\log N\left(R_{0}\right) \\
\left(\mathrm{cm}^{-2}\right)\end{array}$} & \multirow[t]{2}{*}{$N\left(R_{0}\right) / N\left(R_{1}\right)$} & \multirow{2}{*}{$\begin{array}{l}T_{01} \\
(\mathrm{~K})\end{array}$} \\
\hline & & $R_{0}$ & $R_{1}$ & $P_{1}$ & & & \\
\hline \multirow[t]{2}{*}{+6} & $\Sigma$ & $87 \pm 6$ & $36 \pm 4$ & $18 \pm 4$ & $13.44 \pm 0.06$ & & \\
\hline & 1 & $70 \pm 5$ & $29 \pm 3$ & $15 \pm 3$ & $13.37 \pm 0.04$ & 2.14 & $2.9 \pm 0.4$ \\
\hline \multirow[t]{2}{*}{+11} & $\Sigma$ & $79 \pm 6$ & $34 \pm 4$ & $17 \pm 4$ & $13.37 \pm 0.06$ & & \\
\hline & 1 & $63 \pm 4$ & $27 \pm 3$ & $14 \pm 3$ & $13.30 \pm 0.04$ & 1.95 & $3.0 \pm 0.5$ \\
\hline \multirow[t]{2}{*}{+17} & $\Sigma$ & $82 \pm 8$ & $36 \pm 7$ & $14 \pm 7$ & $13.40 \pm 0.08$ & & \\
\hline & 1 & $66 \pm 6$ & $29 \pm 6$ & $11 \pm 5$ & $13.33 \pm 0.06$ & 1.95 & $2.8 \pm 0.6$ \\
\hline
\end{tabular}

Notes. Two velocity components with identical width of $0.11 \AA$ are assumed to contribute to the total absorption profile. This corresponds to an intrinsic FWHM of $5.7 \mathrm{~km} \mathrm{~s}^{-1}$ (for resolution of $6.3 \mathrm{~km} \mathrm{~s}^{-1}$ ) and hence a $b$ of $3.4 \mathrm{~km} \mathrm{~s}^{-1}$. The equivalent width and column density are given for the total line profile and the strongest velocity component, respectively. The average $\mathrm{CN}$ excitation temperature derived from the $R_{0} / R_{1}$ and $R_{0} / P_{1}$ ratios and the $R_{0} / R_{1}$ column density ratios are given for the strongest velocity component only.

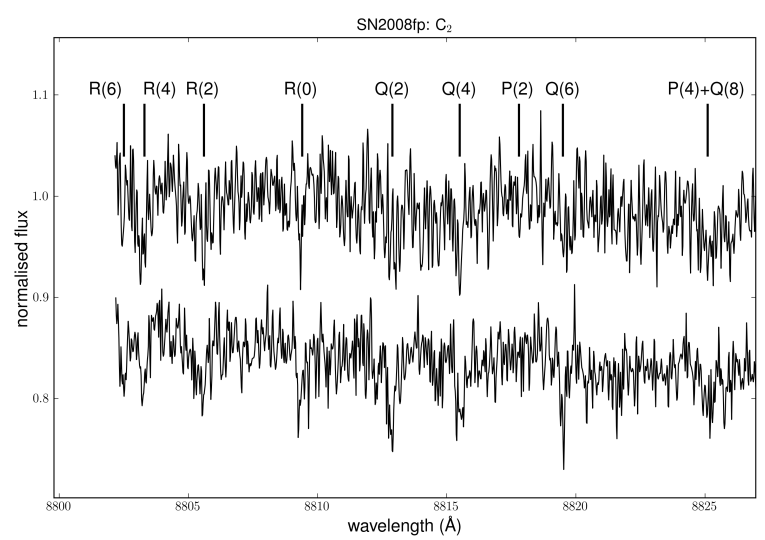

Fig. 3. $C_{2}$ spectrum observed toward SN 2008fp. The indicated line transitions are plotted at a common radial velocity of $1770 \mathrm{~km} \mathrm{~s}^{-1}$, corresponding to the velocity of atomic and di-atomic lines associated to the host galaxy.

with $f=0.0030)$. The ratio $N(\mathrm{CN}(J=0) / N(\mathrm{CN}(J=1)=1.9$, indicating the $\mathrm{CN}(J=0)$ line is weakly saturated (c.f. Sect. 4.6).

$\mathrm{C}_{2}$. The transitions of the $\mathrm{C}_{2}(2,0)$ band detected at velocities of the SN 2008fp host galaxy are only shown for epochs 1 and 2 because only these are included in the UVES Red2 setting (Fig. 3). The indicated line transitions are plotted at a common radial velocity of $1770 \mathrm{~km} \mathrm{~s}^{-1}$. A rotational diagram analysis of the level population of $\mathrm{C}_{2}{ }^{2}$ (see also Sonnentrucker et al. 2007) shows that the interstellar cloud probed by SN 2008fp has particle density $n_{\text {collisions }} \approx 250 \mathrm{~cm}^{-3}$ and rotational temperature $T_{\text {rot }} \approx 30 \mathrm{~K}$.

C . The $\mathrm{C}_{3} Q$-branch $\left(\lambda_{\text {rest }}=4051.6 \AA\right.$; Maier et al. 2001) is detected at a radial velocity of $1768.0 \mathrm{~km} \mathrm{~s}^{-1}$ with an equivalent width of $20 \pm 5 \mathrm{~m} \AA$. The observed $C_{3}$ spectrum is shown in Fig. 4 together with the $\mathrm{C}_{3}$ spectrum of HD $204827\left(T_{\mathrm{C}_{3}} \sim\right.$ $42 \mathrm{~K}$; Ádámkovics et al. 2003). Note the striking resemblance in profile shape and strength of the $Q$-branch peak at $4051.6 \AA$ in the two sightlines. From detailed modeling, Ádámkovics et al. (2003) found that for high gas densities both $\mathrm{C}_{2}$ and $\mathrm{C}_{3}$

2 The web-tool (http://dib.uiuc.edu/c2/calc.html) provided by B.J. McCall was used to infer gas density and temperature from the measured $\mathrm{C}_{2}$ column densities and to correct the observed column density $(J=0-6)$ to obtain the total $(J \leq 20)$ column density. 


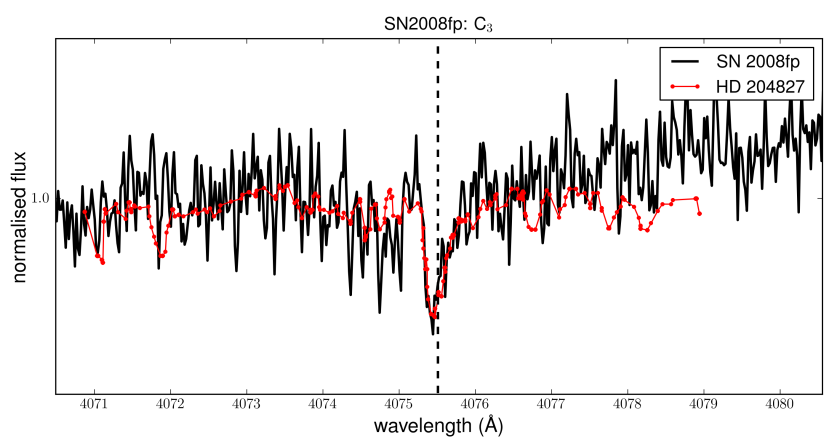

Fig. 4. $C_{3}$ spectrum (black) observed toward SN 2008fp. The vertical dashed line indicates a radial velocity of $1768 \mathrm{~km} \mathrm{~s}^{-1}$. The normalised $\mathrm{C}_{3}$ spectrum of HD 204827 (Ádámkovics et al. 2003), shifted by $1768 \mathrm{~km} \mathrm{~s}^{-1}$, is shown for comparison.

excitation profiles are determined primarily by the kinetic temperature. In other words, the high-J levels of $\mathrm{C}_{3}$ are not significantly populated in dense environments. Indeed, the low population of $R$-band levels, including the clear absence of the $4049.8 \AA$ $R$-band head, is indicative of a thermal distribution at $\sim 50 \mathrm{~K}$ (Ádámkovics et al. 2003). In this case, the approximation that the observed $Q$-branch accounts for about half the total intensity of the $\mathrm{C}_{3}$ band is valid (Oka et al. 2003). Thus, assuming an optically thin line, $N\left(\mathrm{C}_{3}\right)=(17 \pm 5) \times 10^{12} \mathrm{~cm}^{-2}$, similar to $N\left(\mathrm{C}_{3}\right)=(11.51 \pm 0.87) \times 10^{12} \mathrm{~cm}^{-2}$ derived for HD 204827 by Âdámkovics et al. (2003). This result adds to the recent detection of extra-galactic $\mathrm{C}_{3}$ in the SMC towards the peculiar line-of-sight of Sk 143 (Welty et al. 2013) with reported $N\left(\mathrm{C}_{3}\right)=(5.7 \pm 0.6) \times 10^{12} \mathrm{~cm}^{-3}$.

DIBs. The diffuse bands at 5780, 5797, 5849, 6196, and $6283 \AA$ are detected at radial velocities corresponding to the atomic and molecular species reported above. We note that because of the radial velocity shift of the host galaxy ISM, the strong DIB at $6613 \AA$ is expected to be situated just outside the observed spectral range. Radial velocities and equivalent widths are given in Table 3.

\subsubsection{Extra-galactic dust: interstellar polarisation}

Additional and independent information on the nature of the intervening material can be derived by analysing the continuum interstellar linear polarisation (ISP) produced by dust along the line of sight (Serkowski et al. 1975). From the previous section it is clear that SN 2008fp suffers from substantial visual extinction. It is therefore natural to expect a marked interstellar polarisation due to (interstellar) dust, as in the case of SN 2006X (Patat et al. 2009).

We obtained spectropolarimetry of SN 2008fp on days $-5,0$, +2 and +6 days from $B$ maximum light. The data were obtained using FORS1 at the ESO-VLT and were reduced and analysed as described in Patat et al. (2009). Details on the observations will be given in a separate paper, which will also present a detailed study of the intrinsic polarisation properties of SN 2008fp. Here we focus on the ISP in connection with the ISM properties we derived from high-resolution spectroscopy. The data show a continuum polarisation that steadily grows from the red to the blue, exceeding $2.2 \%$ at $4200 \AA$ (Fig. 5). The polarisation wavelength dependency shows no signs of the maximum typically seen in highly reddened Galactic stars, which occurs on average around

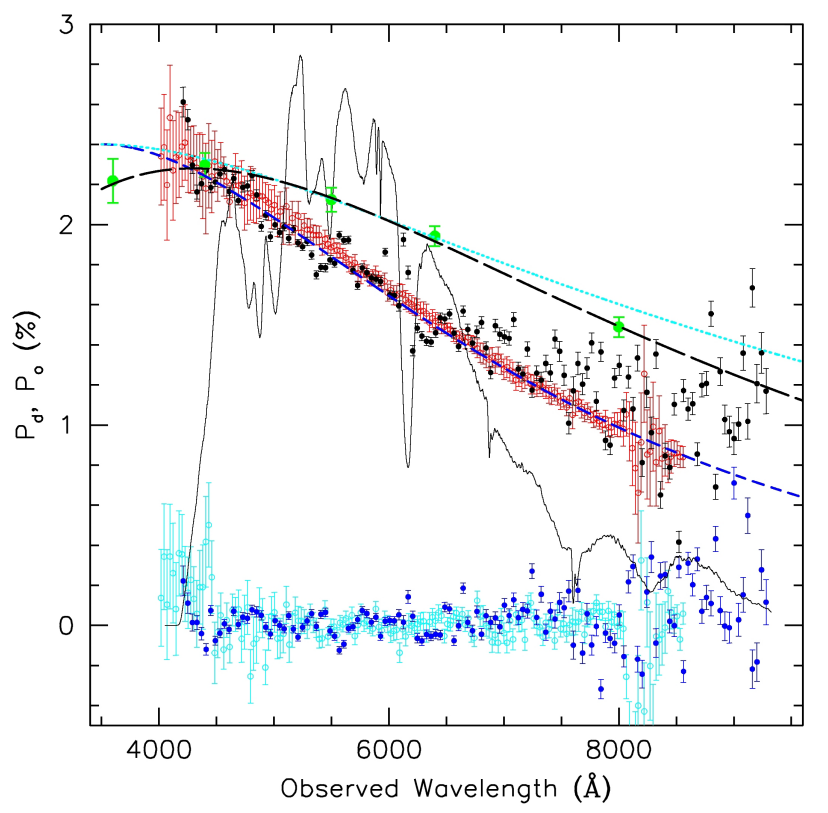

Fig. 5. Spectropolarimetry of SN 2008fp along the dominant (upper) and orthogonal (lower) axes (filled symbols) on day +6 . The underlying solid curve traces the unbinned observed spectrum of SN 2008fp on the same epoch. For comparison the scaled data of SN 2006X are presented as well (empty symbols; Patat et al. 2009). The short-dashed line is a best fit to the SN 2006X data using a Serkowski law $\left(\lambda_{\max }=3500 \AA\right.$, $K=1.30$ ), while the dotted line is the same but with $K$ given by the Whittet et al. (1992) relation $(K=0.59)$. The large, filled dots indicate the scaled broad-band polarimetry measurements of SN 1986G (Hough et al. 1987). The long-dashed curve traces a best-fit Serkowski law $\left(\lambda_{\max }=4300 \AA\right.$, $\left.K=1.10\right)$.

$5400 \AA$ A (Serkowski et al. 1975; Whittet et al. 1992). The continuum polarisation angle measured on the combined spectrum is $148.4 \pm 0.15$ degrees. This is very well aligned to the local spiral structure, as in other well-observed SNe and consistent with the orientation of dust grains along the Galactic magnetic field (Scarrott et al. 1987).

However, the derivation of ISP in SN 2008fp is made somewhat uncertain by two problems: i) the ISP appears to be more than a factor four smaller than in the extreme case of $2006 \mathrm{X}$ (Patat et al. 2009); ii) no late-time polarimetric data are available. This implies that the SN imprints are expected to be more marked in 2008fp than in 2006X. Furthermore, the data suffer from a marked fringing redwards of $6000 \AA$, increasing the noise level well above the photon noise statistics in that spectral region. This is illustrated in Fig. 5, where we plot the decomposition of the observed Stokes parameters $Q$ and $U$ along the dominant (identified by the global polarisation angle) and the orthogonal axis, $P_{d}$ and $P_{O}$, respectively. To minimise the SN contribution to the observed polarisation we chose to use the last available epoch (day +6). As anticipated, SN 2008fp displays a pronounced continuum polarisation along the dominant direction (148.4 degrees), on top of which some intrinsic features are detected at the positions of the main absorptions of the flux spectrum, chiefly Si II $6355 \AA$ A and Ca II NIR triplet. While signatures of these features are also visible in the orthogonal component, the continuum polarisation is consistent with zero in the wavelength range 4000-7500 $\AA$. At redder wavelengths there may be an additional continuum contribution, although this region is affected both by the Ca II triplet and by fringing, most likely responsible for the observed scattering. The "residual" continuum 
polarisation is below the $0.2 \%$-level, which is typical of normal Type Ia (Patat et al. 2009). The comparison between polarisation curves of SN 2008fp and SN 2006X is discussed in Sect. 4.8.

\section{Discussion}

In this section we discuss the properties of the extra-galactic ISM in the line-of-sight of SN 2008fp.

\subsection{Extra-galactic diffuse interstellar bands}

The $5780 \AA$ DIB correlates with $E_{(B-V)}$ in the MW, MCs, and DLA systems. Thus, DIB formation/survival and high dust content are closely linked. Several surveys have found that the $5780 \AA$ DIB is, for the same H I column density, typically a factor of 8 and 20 weaker in the LMC and SMC than in the Milky Way (Cox et al. 2006; Welty et al. 2006). Moreover, the $5780 \AA$ DIB is three to six times weaker in DLAs for a given $N(\mathrm{HI})$ than in the MW sightlines, whereas the 6283 is $4-10$ times under-abundant (York et al. 2006; Lawton et al. 2008). The observed $5780 \AA$ DIB strength is consistent with the Galactic $E W$ $E_{(B-V)}$ relation.

The equivalent widths of the 5780, 5797, 5849, and $6196 \AA$ DIBs predict, assuming a Galactic trend (Luna et al. 2008), an $E_{(B-V)}$ between 0.20 and $0.55 \mathrm{mag}$. At low reddening values the scatter on these relationships can be as much as a factor of two.

However, a recent survey by Vos et al. (2011) showed that the correlation of the 5780 DIB with reddening is different for $\sigma$ and $\zeta$-type sightlines ${ }^{3}$. For a $\zeta$-type line-of-sight the $5797 \AA$ DIB is deeper than the 5780 DIB. In addition, for SN 2008fp the $5797 / 5780$ strength ratio is 0.74 . Such high values are generally only observed toward dense ( $\zeta$-type) Galactic sightlines. We note that the reduced strength of the "broad" 5780 and 6283 DIBs with respect to those of the "narrow" DIBs at 5797, 5849 and $6196 \AA$ is typical, thus expected, for dense clouds in which the production of the carrier of the former family is apparently less efficient. Figure 6 shows the 5780 and $5797 \AA$ A DIBs together with those towards two Galactic sightlines that are typical of a diffuse cloud exposed to a strong UV field ( $\sigma$-type) and a denser (molecular) cloud shielded from the interstellar UV radiation field ( $\zeta$-type). Scaling the 5780-5797 DIB spectrum observed toward Galactic $\zeta$ type HD $149757\left(E_{(B-V)}=0.32 \mathrm{mag}\right)$ by about $40 \%$ provides a good fit to the two diffuse bands, thus implying that this dense cloud has $E_{(B-V)} \approx 0.45$ mag (Figs. 6 and 1). For $R_{\mathrm{V}} \leq 2$ (Sect. 4.5) this corresponds to $A_{\mathrm{V}} \leq 0.9$ mag.

The narrow profiles of the observed di-atomic molecules and their relative high-abundance together with the inferred high molecular hydrogen fraction and intermediate visual extinction of 0.9 mag indicates that the line-of-sight towards SN 2008fp probes a translucent cloud (Snow \& McCall 2006).

For Galactic sightlines with high abundances of $\mathrm{C}_{2}$ several DIBs are also observed to be stronger with respect to the other DIBs. The strongest $\mathrm{C}_{2}$-DIB is the $\lambda 4963$ band, although the 4734 and $5769 \AA$ DIBs shows the tightest correlation with $N\left(\mathrm{C}_{2}\right) / E_{(B-V)}$ (Thorburn et al. 2003). Unfortunately, the 4963 and $4737 \AA$ DIBs (at the host galaxy velocity) are not included

\footnotetext{
$3 \sigma$ and $\zeta$-type diffuse clouds refer to the archetypical Galactic linesof-sight $\sigma$ Sco and $\zeta$ Oph. $\sigma$ Sco probe a diffuse environment exposed to a strong radiation field with low molecular content, while $\zeta$ Oph probes a denser cloud protected from the impinging interstellar radition. For a discussion on the two types of clouds and how this connects to molecular content, the ISRF strength and DIB (ratio) we refer to Vos et al. (2011).
}

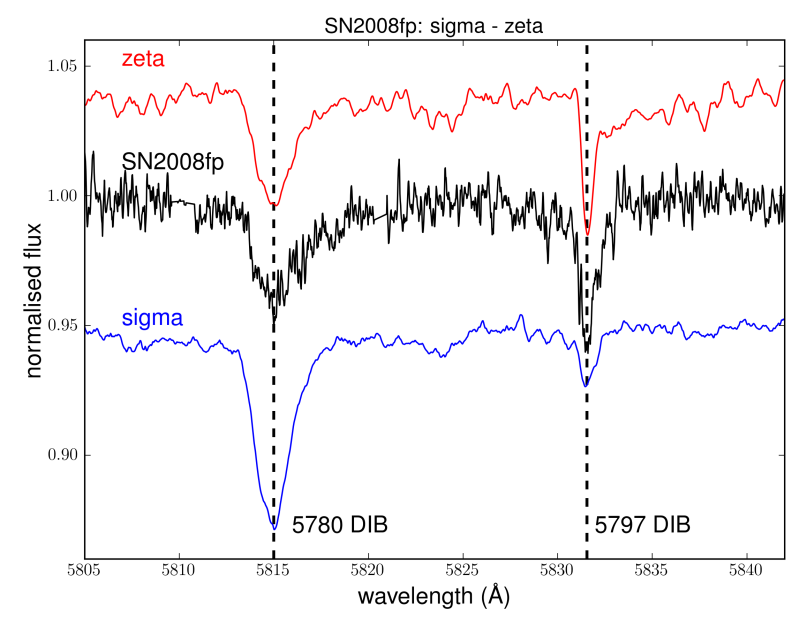

Fig. 6. Extra-galactic 5780 and 5797 DIBs at 1800 and $1789 \mathrm{~km} \mathrm{~s}^{-1}$ towards SN 2008fp. Typical $\sigma$ (HD 144217) and $\zeta$ (HD 149757) Galactic diffuse cloud spectra are shown for comparison (from Vos et al. 2011). The normalised spectra are offset vertically for clarity and the Galatic spectra were shifted in velocity to match the observed DIBs towards SN 2008fp. See the text for more details.

in our spectral range. The $5769 \AA$ DIB was not detected (its enhanced $E W / E_{(B-V)}$ is only $\left.18 \mathrm{m \AA}\right)$.

We note also that the $C_{2}$ near-infrared band ( $J$ levels from 0 to 8) has recently been detected in the Small Magellanic Cloud, thus for the first time beyond the Milky Way, for the peculiar line-of-sight towards Sk 143 (Welty et al. 2013). The detection of $\mathrm{C}_{2}$ and $\mathrm{C}_{3}$ in the SN 2008fp host galaxy constitutes the first direct detection of these molecules beyond the Local Group. These observations illustrate that optical high-resolution spectroscopy of $\mathrm{SNe}$ offers an unique opportunity to probe in detail the physics and chemistry of the diffuse cold medium in distant galaxies. In particular, $\mathrm{C}_{2}$ is a sensitive probe to local density and temperature, but requires both high-resolution and high $\mathrm{S} / \mathrm{N}$ in the optical range, which is currently only accessible for relatively bright sources even with $10 \mathrm{~m}$-class telescopes.

\subsection{Molecular hydrogen fraction}

In this section we attempt to derive an estimate of the molecular hydrogen fraction of the extra-galacitc translucent cloud in the line-of-sight toward SN 2008fp. First, the H I column density can be estimated from the 5780 DIB strength: $\log (N(\mathrm{HI})=(19.00 \pm$ $0.08)+(0.94 \pm 0.04) \log (W(5780))($ Friedman et al. 2011), which gives for SN 2008fp $N(\mathrm{HI})=6.2_{-2.1}^{+3.3} \times 10^{20} \mathrm{~cm}^{-2}$. Then, for Galactic sightlines, the $\mathrm{CH}$ column density shows a strong linear relation with the $\mathrm{H}_{2}$ column density: $N(\mathrm{CH}) / N\left(\mathrm{H}_{2}\right)=$ $3.5_{-1.4}^{+2.1} \times 10^{-8}$ (see e.g. Federman 1982; Sheffer et al. 2008). Thus, $N\left(\mathrm{H}_{2}\right)=7.2_{-2.7}^{4.8} \times 10^{20} \mathrm{~cm}^{-2}$. Now we obtain $f_{\mathrm{H}_{2}}=$ $2 N\left(\mathrm{H}_{2}\right) /\left(N(\mathrm{HI})+2 N\left(\mathrm{H}_{2}\right)\right)=0.70_{-0.20}^{+0.15}$. This confirms our earlier conclusion that the line-of-sight towards SN 2008fp probes a dense, $\zeta$-type cloud in the host galaxy.

\subsection{Carbon chemistry and abundance}

The ratio $\mathrm{CH}^{+} / \mathrm{CH}$ scales with the strength of the interstellar radiation field $\left(I_{\mathrm{UV}}\right)$, density $\left(n_{\mathrm{H}}\right)$ and molecular hydrogen fraction $\left(f_{\mathrm{H}_{2}}\right)$ as derived by e.g. Welty et al. (2006) and Ritchey et al. (2006): $N\left(\mathrm{CH}^{+}\right) / N(\mathrm{CH}) \propto I_{\mathrm{UV}} /\left(n_{\mathrm{H}} f_{\mathrm{H}_{2}}\right)$. The measured ratio, $N\left(\mathrm{CH}^{+}\right) / N(\mathrm{CH})=0.6_{-0.44}^{+1.7}$, is similar to that observed toward SN 2006X (Cox \& Patat 2008). It is indicative of inefficient $\mathrm{CH}^{+}$production and thus a quiescent ISM 
(e.g. Crawford 1989; Vos et al. 2011). However, since the (average) density and molecular fraction are high (typical for translucent clouds), the ambient interstellar UV radiation field can be relatively strong despite the low ratio $\mathrm{CH}^{+} / \mathrm{CH}$.

The observed ratio $N\left(\mathrm{C}_{2}\right) / N\left(\mathrm{C}_{3}\right)$ is $12 \pm 8$. This ratio is, within the uncertainties, similar to mean ratio of $28.5 \pm 6.0$ (ranging from 7.6 to 68.1) for a sample of 13 Galactic sightlines (Ádámkovics et al. 2003). Note also that the observed total column densities for $\mathrm{C}_{2}$ towards SN 2008fp is similar to that measured for the Galactic sightlines HD 204827, which has the highest measured $\mathrm{C}_{2}$ and $\mathrm{C}_{3}$ columns density in the survey by Ádámkovics et al. (2003). The observed $\mathrm{C}_{3}$ column density is about 50\% higher towards SN 2008fp, which results in a lower ratio than the ratio of 38.3 for HD 204827.

Adopting the values above, the host galaxy ISM displays a high carbon fraction, $f\left(\mathrm{C}_{2}\right)=N\left(\mathrm{C}_{2}\right) / N\left(\mathrm{H}_{\text {total }}\right)=9.7 \times 10^{-8}$, which is a factor two higher than the Galactic fraction, $f\left(\mathrm{C}_{2}\right)_{\mathrm{MW}}=3-6 \times 10^{-8}$, found by Van Dishoeck \& Black (1989) for diffuse clouds $\left(A_{\mathrm{V}}<5 \mathrm{mag}\right)$, and a factor six higher than values found by Gredel (1999), $f\left(\mathrm{C}_{2}\right)_{\mathrm{MW}}=0.5-1.5 \times 10^{-8}$.

\subsection{Metal depletion}

Taking the tentative detection of Ti II at face value we obtain, together with $N\left(\mathrm{H}_{\text {total }}\right)$, the gas phase interstellar abundance of titanium. Since the ionisation potential of Ti II is nearly coincident to that of $\mathrm{HI}$, Ti II is the dominant ion in $\mathrm{HI}$ regions (see e.g. Welty \& Crowther 2010). Most of the titanium in the Galactic ISM is depleted into dust grains with less than 1 percent of the total titanium in the gas phase (e.g. Jenkins 2009). Like Ca II, Ti II traces warmer, more smoothly distributed neutral component of the ISM (Crinklaw et al. 1994). The ratio $N$ (Ti II)/N(Ca II) is fairly constant, with a value of $0.3-0.4$ for Galactic environments (Albert 1983; Hunter et al. 2006), but is on average higher, at about 0.9 , for the SMC and LMC. Welty \& Crowther (2010) showed that there is no significant saturation for the Ti II $3383.768 \AA$ line up to $100 \mathrm{~m} \AA$. For SN 2008fp the observed ratio of $N(\mathrm{Ti}$ II $) / N(\mathrm{Ca}$ II $)$ is 0.05 , and the relative depletion $\mathrm{Ti} / \mathrm{H}$ is $-9.2 \mathrm{dex}$, compared with -7.1 dex for the solar neighbourhood. Taken at face value, this would imply that metals appear to be heavily depleted into dust grains in the dense extragalactic line-of-sight towards SN 2008fp. However, given the uncertainties in the different column densities and abundances, we caution against drawing any firm conclusions.

Cardelli et al. (1991) found that $N(\mathrm{CaI}) / N(\mathrm{KI}) \sim \delta(\mathrm{Ca})$, where the calcium depletion $\delta(\mathrm{Ca})$ is $\propto n_{\mathrm{H}}^{3}$. For SN 2008fp the measured ratio of $0.214_{-0.16}^{1.74}$ is low, which gives $\delta(\mathrm{Ca}) \sim$ $0.017-0.028$ (or $\log \delta(\mathrm{Ca}) \stackrel{-0.16}{\sim}-1.6$ to -1.8 ), depending on whether photoionisation equillibrium or charge exchange processes are assumed. In Galactic environments low ratios are seen for clouds with molecular hydrogen fractions $>0.1$.

\subsection{Dust polarisation properties}

The polarisation wavelength dependency shown by SN 2008fp is reminiscent of what was found in SN 2006X (Patat et al. 2009). This is illustrated in Fig. 5 where, for presentation, the dominant axis component was scaled by a factor 3.3 to match the data-sets of the two $\mathrm{SNe}$ at $\sim 4000 \AA$. Bearing in mind that in SN 2008fp the intrinsic SN signatures are stronger, the resemblance is significant. In both cases the polarisation grows at shorter wavelengths and, if anything, the maximum is attained below $4000 \AA$. As described in Patat et al. (2009), the polarisation wavelength dependency of SN 2006X can be reproduced by a Serkowski law with $\lambda_{\max }=3500 \pm 100 \AA$ and $K=1.3 \pm 0.1$ (see Fig. 5, short-dashed line). The value of $K$ is higher than $K=1.15$ derived by Serkowski et al. 1975) and disagrees with the wavelength dependency derived by Whittet et al. (1992), $K=0.01+1.66 \lambda_{\max }$ (where $\lambda_{\max }$ is expressed in $\mu \mathrm{m}$ ), yielding $K=0.60$ for $\lambda_{\max }=0.35 \mu \mathrm{m}$. The corresponding polarisation law is traced in Fig. 5 (dotted line), which clearly illustrates the poor match to the data.

Hough et al. (1987) pointed out that $\lambda_{\max }$ for SN 1986G is lower than typical MW values, while it is closer to what is found in M31. Assuming that the dust composition is similar, they concluded that the grain sizes must be about $20 \%$ smaller in Cen A than in the MW and postulated that this should have some consequences on the exctinction law. Using the empirical relation derived by Serkowski et al. (1975) between the selective-to-total absorption $R_{\mathrm{V}}$ and $\lambda_{\max }, R_{\mathrm{V}}=(5.6 \pm 0.3) \lambda_{\max }$, they derived $R_{\mathrm{V}}=2.4 \pm 0.13$ for $\mathrm{SN} 1986 \mathrm{G}$. This value agrees remarkably well with the photometric derivation: $R_{\mathrm{V}}=2.6 \pm 0.2$ (Phillips et al. 2013).

The cases of SN 2006X and SN 2008fp appear to be even more extreme. As pointed out in Patat et al. (2009), the lack of a maximum polarisation in SN 2006X is a convincing argument in favour of an exceptionally small $R_{\mathrm{V}}$, giving independent support to the photometric derivations: $1.48 \pm 0.06$ (Wang et al. 2008), 1.31 $1_{-0.10}^{+0.08}$ (Phillips et al. 2013). Given the spectropolarimetric data presented here, we argue that this plausibly applies to SN 2008fp as well. Using $\lambda_{\max } \leq 3500 \AA$ in the above relation gives $R_{\mathrm{V}} \leq 2$, consistent with $R_{\mathrm{V}}=1.20_{-0.14}^{+0.26}$ derived from the light curve (Phillips et al. 2013). Along the lines proposed by Hough et al. (1987), we conclude that the dust grains composition and/or size must be substantially different from those typical of the MW (see also Whittet 1992).

One possible problem with the ISP determination for SN 2008fp is the unaccounted MW contribution. The Galactic foreground reddening is $E_{(B-V)}=0.2$ (Schlegel et al. 1998). The host galaxy photometric reddening estimate is $E_{(B-V)}=$ $0.6 \pm 0.1$, implying that the Galactic reddening contribution is about one forth of the total. In the above discussion we implicitly assumed that the MW contribution is not significant. This is supported by the position angle alignment with the host galaxy spiral pattern. However, we cannot exclude a contribution to the total polarisation of the order of a several $0.1 \%$, depending on the angle between the host and Galactic polarisation angles.

Given the findings of high-resolution spectroscopy, we conclude that the bulk of reddening, and therefore of polarisation, occurs within the molecular material. Whether or not this is related to the progenitors cannot be established based on the data presented here. However, given the projected positions of the two events in their hosts, the most natural explanation is that the intervening gas belongs to the host galaxy ISM. Irrespective of the location of this material, it remains to be clarified why in these two objects the dust displays properties that deviate so extremely from the typical MW mixture. The question as to how this is related to the growing evidence about Type Ia SN showing systematically small $R_{\mathrm{V}}$ has to be addressed using statistically significant samples that now start to be available (Zelaya et al., in prep.).

\subsection{Cosmic radiation background}

The relative line strengths of the $R_{0}$ and $R_{1}$ absorption lines can be used to derive the number of $\mathrm{CN}$ molecules in the ground- and 
first-excited rotational states, with which one can determine the excitation temperature of the $J=0-1$ transition at $2.64 \mathrm{~mm}$ (Thaddeus 1972; Ritchey et al. 2011; Leach 2012). In the absence of local excitation, $T_{01}=(1+z) T_{\mathrm{CMB}}=2.7416 \pm 0.003 \mathrm{~K}$ $\left(T_{\mathrm{CMB}-\mathrm{COBE}}=2.72548 \pm 0.00057 \mathrm{~K}\right.$; Mather et al. 1999; Fixsen 2009) and thus $N\left(R_{0}\right) / N\left(R_{1}\right)=2.463$. Note that Meyer \& Jura (1985) showed that local collisional excitation of CN by electrons increases $T_{01}$ by only $0.2 \mathrm{~K}$ even for a high electron density, $n_{\mathrm{e}}=0.5 \mathrm{~cm}^{-3}$.

Since both $R_{1}$ and $P_{1}$ arise from the first excited rotational state of $\mathrm{CN}$, we can use both features, together with $R_{0}$, to derive $T_{01}$. Here we assumed that the observed profile is a composite of at least two individual velocity components with $b \sim$ $3.4 \mathrm{~km} \mathrm{~s}^{-1}$, derived from decomposing the observed line profile into two components (separated by $\sim 9.6 \mathrm{~km} \mathrm{~s}^{-1}$ ) of equal intrinsic FWHM of $5.7 \mathrm{~km} \mathrm{~s}^{-1}$. With the present data we cannot exclude the presence of unresolved narrow $\left(b \approx 1 \mathrm{~km} \mathrm{~s}^{-1}\right)$ velocity components such as those revealed by high-resolution spectroscopic studies of Galactic translucent clouds (e.g. Meyer \& Jura 1985; Gredel et al. 1991; Pan et al. 2005; Słyk et al. 2008).

Next, we applied the curve-of-growth correction to the strongest velocity component following the method described by Meyer \& Jura (1985) (the measurements of the weak redshifted component are of insufficient quality to derive useful quantities). The results are listed in Table 4 . The mean excitation temperature derived from the line ratios $R_{0} / R_{1}$ and $R_{0} / P_{1}$ is $T_{01}=2.9 \pm 0.4 \mathrm{~K}$ for the first epoch. In addition, we obtain $N\left(R_{0}\right) / N\left(R_{1}\right)=2.1$. Spectra taken at the second and third epoch are consistent with the first epoch result, but give larger uncertainties. We note that although the absolute excitation temperature is sensitive to the adopted $b$, the difference in derived temperatures is much less sensitive. Reducing $b$ to $3.0 \mathrm{~km} \mathrm{~s}^{-1}$ reduces the temperature to $T_{01}=2.8 \mathrm{~K}$. Within the uncertainties on the line measurements there is no evidence for significant local excitation of $\mathrm{CN}$ in addition to the excitation provided by the cosmic microwave background, which is the main reservoir of $2.64 \mathrm{~mm}$ photons.

\subsection{Line variability}

The UVES data span from about 2 days before maximum to 30 after maximum, which translates into a time-coverage of 17 to 50 days after the estimated explosion (assuming the canonical 19-day rise-time of Type Ia SN). In this time span the photosphere expanded approximately from $120 \mathrm{AU}$ to $240 \mathrm{AU}$, that is, doubling its size. In the case of a patchy ISM with a fractal structure (Patat et al. 2010), the peak-to-peak variations in this time range are always smaller than a few tens of $m \AA$, with the rms variations being of the order of a few $m \AA$. Therefore, the lack of larger variations, for example the $\mathrm{CN}$ line measurements at phases 1 and 2, excludes small, isolated clouds (or dense compact cloudlets) with sizes similar to the $\mathrm{SN}$ photospheric radius (100-200 AU). A detailed discussion of the (lack of) variability in column denstities of the atomic $\mathrm{NaI}$ and $\mathrm{Ca}$ II lines will be presented elsewhere as part of a larger survey studying the variability in atomic absorption profiles towards Type Ia supernovae.

\subsection{Probing extra-galactic molecular clouds: SN2006X and SN2008fp}

Excitation analyses of the $\mathrm{UV} /$ blue $\mathrm{CN}(0,0)$ band in $\mathrm{SNe}$ host galaxies offer a first step towards an in situ probe of the CMB temperature history, as suggested by Weiss (1980). For
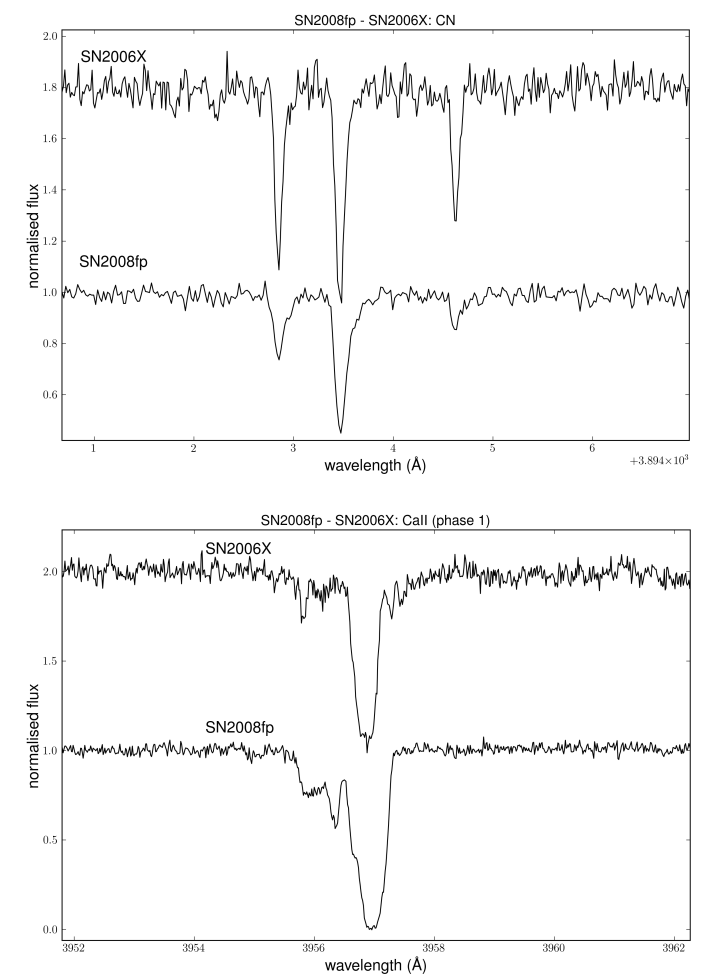

Fig. 7. CN and Ca II line profiles for both SN 2008fp and SN 2006X are shown, both at 2 days before maximum light. The unresolved CN lines in SN 2006X do not reveal the red wing seen for SN 2008fp.

now this has been possible for relatively nearby SNe 2006X and 2008fp, which have redshifts of $z=0.005240$ and $z=$ 0.005664 , respectively. We note that for SN 2006X the CN excitation temperatures, $T_{\mathrm{ex}}=3.0 \pm 0.2 \mathrm{~K}$, is also fully consistent with the CMB (Patat et al. 2007).

For SN 2006X the $E_{(B-V)}$ was estimated to be around 1.2 to 1.4 mag (see e.g. Patat et al. 2009). It appears that SN 2008fp suffers a lower extinction of $E_{(B-V)} \approx 0.45 \mathrm{mag}$. SN 2008fp is located at a projected distance of about $2.2 \mathrm{kpc}$ from the host galaxy nucleus, and from its axial ratio one deduces an inclination of about 55 degrees. Its relatively high extinction furthermore suggests that it is placed at a low galactic latitude or behind the disk of the host galaxy. This is similar to the position of SN 2006X in its host galaxy.

As discussed above, the extinction measured towards both $\mathrm{SNe}$, in total intensity and polarised light, is primarily due to the ISM and not any CSM. Thus a direct comparison of the CN and $\mathrm{Ca}$ II line profiles presents itself rather naturally (Fig. 7). Although similar, the small difference in line profile of the individual transitions further substantiates that for SN 2008fp we observe two components in $\mathrm{CN}$, instead of it having a higher $b$-value. The observed similarity with SN 2006X in terms of CN and extinction, but lack of a variable sodium component, clearly shows that high extinction does not necessarily imply time variations, as noted already by Simon et al. (2009). At this time we cannot pinpoint the origin of the unusually strong $\mathrm{CN}$ lines towards these two $\mathrm{SNe}$, although it appears to be related somehow to a specific subset of Type Ia SNe and/or their host galaxies. Another recent example is SN2009ig, which also shows strong $\mathrm{CN}$ absorption lines relative to the line-of-sight visual extinction (Cox \& Patat 2014).

At face value, the ratio between the $E_{(B-V)}$ of SN 2006X and $\mathrm{SN} 2008 \mathrm{fp}$ is $\approx 2.7-3.1$, close to the polarisation ratio of 3.3 , 
implying that SN 2008fp has a polarisation that is 10-20\% lower than one would expect if it behaved like 2006X. This can be interpreted as a slightly lower efficiency of grain alignment in the intervening material of SN2008fp, which suffers a reddening higher than its polarisation would indicate, although the effect is minimal. One possibility is that this is related to the anomalous ratio between the $\mathrm{NaID}$ equivalent width and reddening (Phillips et al. 2013).

\section{Conclusion}

We presented deep high-resolution optical spectra of the Type Ia supernovae $2008 \mathrm{fp}$. We focused on the analysis and interpretation of these spectra in the context of understanding the physical properties of interstellar molecular clouds in extra-galactic environments. This analysis of the line-of-sight towards SN 2008fp revealed the following:

- In addition to the main atomic species ( $\mathrm{Na}$ I, Ca II), many weaker lines are detected: Fe I, Ca I, Ti II, $\mathrm{CH}, \mathrm{CH}^{+}, \mathrm{CN}$, as well as diffuse interstellar bands at 5780, 5797, 5849, 6196, and $6283 \AA$.

- The 5780 and $5797 \AA$ DIBs indicate that the $\zeta$-type cloud component at $1770 \mathrm{~km} \mathrm{~s}^{-1}$ can be considered a translucent cloud sufficiently shielded from interstellar UV radiation (from young stars in the host galaxy) to ensure a rich molecular chemistry. From a comparision with Galactic $\sigma$ and $\zeta$-type DIB spectra we inferred $E_{(B-V)}=0.45 \mathrm{mag}$ for the total extra-galactic reddening towards SN 2008fp. This fully agrees with $A_{\mathrm{V}}$ and $R_{\mathrm{V}}$ derived from modelling the light curve.

- The molecular hydrogen fraction of $0.7_{-0.2}^{0.15}$ corresponds to that of translucent clouds.

- The $\mathrm{C}_{2}(2,0)$ band is detected beyond the Local Group, in the SN 2008fp host galaxy. The rotational line analysis yielded $T_{\text {rot }} \approx 30 \mathrm{~K}$ and $n_{C} \approx 250 \mathrm{~cm}^{-3}$. The $\mathrm{C}_{2} / \mathrm{H}_{\text {total }}$ fraction is a factor three or more higher than typically found for Galactic diffuse clouds.

- We presented a column density of $N\left(\mathrm{C}_{3}\right)=1.7 \pm 0.5 \times$ $10^{13} \mathrm{~cm}^{-2}$ for extra-galactic $\mathrm{C}_{3}$ towards SN 2008fp, which constitutes the first detection of $\mathrm{C}_{3}$ beyond the Local Group. The relative abundance of $C_{3}$ with respect to $C_{2}$ is similar to that of the Galactic mean.

- The relative depletion of $\mathrm{Ti} / \mathrm{H}$ is $\approx-9.2 \mathrm{dex}$, which indicates a higher level of depletion than the -7.1 dex depletion found for the solar neighbourhood.

- From the atomic and molecular line analysis we conclude that the bulk of reddening (dust extinction), and therefore of polarisation, occurs within molecular clouds in the SN 2008fp host galaxy along the line of sight. If not physically associated with the SN progenitor, this implies that the reddening and polarisation arise primarily in the ISM and not in the CSM. This conclusion is strengthened by the polarisation position angle. If the dust were of CS nature, it would produce polarisation by scattering, which would turn into a non-null net polarisation only if the dust is distributed asymmetrically.

- The wavelength dependency of interstellar polarisation in the host of SN 2008fp clearly differs from that displayed by similarly reddened stars in the Galaxy. The observed behaviour is very similar to that of SN 2006X, which also shows a total-to-selective extinction ratio $R_{V}<2$, indicating a dust size/composition significantly different from that typical of the Milky Way.
- From the $\mathrm{CN}(0,0)$ band the $\mathrm{CN}$ excitation temperature at $2.64 \mathrm{~mm}$ is $2.9 \pm 0.4 \mathrm{~K}$, consistent with that of the cosmic microwave background, and statistically inconclusive regarding local collisional excitations by electrons.

- The lack of variability over a 28-day period excludes small, isolated clouds with sizes similar to the SN photospheric radius of 100 to $200 \mathrm{AU}$, supporting the presence of a more patchy - fractal - ISM.

- High-resolution spectra of Type Ia SNe reveal several sightlines - SN 2006X, SN 2008fp, SN 2009ig - with an inexplicably strong CN B-X $(0,0)$ absorption band. Whether and how this is related to a specific subset of Type Ia SNe or their host galaxies remains an open question.

Acknowledgements. We thank the referee for a careful reading of the manuscript and constructive insights that improved this paper. We have benefitted from discussions with Dan Welty on the topic of molecular optical spectroscopy. This research has made use of NASA's Astrophysics Data System Bibliographic Services, of the NASA/IPAC Extragalactic Database (NED) which is operated by the Jet Propulsion Laboratory, California Institute of Technology, under contract with the National Aeronautics and Space Administration, and of the SIMBAD database, operated at CDS, Strasbourg, France.

\section{References}

Ádámkovics, M., Blake, G. A., \& McCall, B. J. 2003, ApJ, 595, 235 Albert, C. E. 1983, ApJ, 272, 509

Blondin, S., Prieto, J. L., Patat, F., et al. 2009, ApJ, 693, 207 Bowen, D. V., Roth, K. C., Blades, J. C., \& Meyer, D. M. 1994, ApJ, 420, L71

Cardelli, J. A., Federman, S. R., \& Smith, V. V. 1991, ApJ, 381, L17 Cordiner, M. A., Cox, N. L. J., Trundle, C., et al. 2008a, A\&A, 480, L13 Cordiner, M. A., Smith, K. T., Cox, N. L. J., et al. 2008b, A\&A, 492, L5 Cox, N. L. J., \& Patat, F. 2008, A\&A, 485, L9

Cox, N. L. J., \& Patat, F. 2014, in IAU Symp. 297, eds. J. Cami, \& N. L. J. Cox, 106

Cox, N. L. J., Cordiner, M. A., Cami, J., et al. 2006, A\&A, 447, 991

Crawford, I. A. 1989, MNRAS, 241, 575

Crinklaw, G., Federman, S. R., \& Joseph, C. L. 1994, ApJ, 424, 748

De Vaucouleurs, G., De Vaucouleurs, A., Corwin Jr., H., et al. 1991, third reference catalogue of bright galaxies, version 3.9 (RC3)

D’Odorico, S., Pettini, M., \& Ponz, D. 1985, ApJ, 299, 852

D'Odorico, S., di Serego Alighieri, S., Pettini, M., et al. 1989, A\&A, 215, 21

Ellison, S. L., York, B. A., Murphy, M. T., et al. 2008, MNRAS, 383, L30

Federman, S. R. 1982, ApJ, 257, 125

Fixsen, D. J. 2009, ApJ, 707, 916

Friedman, S. D., York, D. G., McCall, B. J., et al. 2011, ApJ, 727, 33

Gredel, R. 1999, A\&A, 351, 657

Gredel, R., van Dishoeck, E. F., \& Black, J. H. 1991, A\&A, 251, 625

Grewing, M., Blades, J. C., Panagia, N., Pettini, M., \& Wamsteker, W. 1987, in European Southern Observatory Conference and Workshop Proc. 26, ed. I. J. Danziger, 511

Hough, J. H., Bailey, J. A., Rouse, M. F., \& Whittet, D. C. B. 1987, MNRAS, 227, $1 \mathrm{P}$

Hunter, I., Smoker, J. V., Keenan, F. P., et al. 2006, MNRAS, 367, 1478

Jenkins, E. B. 2009, ApJ, 700, 1299

Junkkarinen, V. T., Cohen, R. D., Beaver, E. A., et al. 2004, ApJ, 614, 658

Lawton, B., Churchill, C. W., York, B. A., et al. 2008, AJ, 136, 994

Leach, S. 2012, MNRAS, 421, 1325

Luna, R., Cox, N. L. J., Satorre, M. A., et al. 2008, A\&A, 480, 133

Maier, J. P., Lakin, N. M., Walker, G. A. H., \& Bohlender, D. A. 2001, ApJ, 553, 267

Mather, J. C., Fixsen, D. J., Shafer, R. A., Mosier, C., \& Wilkinson, D. T. 1999, ApJ, 512, 511

Meyer, D. M., \& Jura, M. 1985, ApJ, 297, 119

Meyer, D. M., \& Roth, K. C. 1991, ApJ, 383, L41

Oka, T., Thorburn, J. A., McCall, B. J., et al. 2003, ApJ, 582, 823

Pan, K., Federman, S. R., Sheffer, Y., \& Andersson, B. 2005, ApJ, 633, 986

Pettini, M. 1988, Proc. Astron. Soc. Aust., 7, 527

Patat, F., Chandra, P., Chevalier, R., et al. 2007, Science, 317, 924

Patat, F., Baade, D., Höflich, P., et al. 2009, A\&A, 508, 229

Patat, F., Cox, N. L. J., Parrent, J., \& Branch, D. 2010, A\&A, 514, A78

Patat, F., Cordiner, M. A., Cox, N. L. J., et al. 2013, A\&A, 549, A62 
Phillips, M. M., Simon, J. D., Morrell, N., et al. 2013, ApJ, 779, 38

Pignata, G., Maza, J., Hamuy, M., et al. 2008, Central Bureau Electronic Telegrams, 1506,1

Rich, R. M. 1987, AJ, 94, 651

Ritchey, A. M., \& Wallerstein, G. 2012, ApJ, 748, L11

Ritchey, A. M., Martinez, M., Pan, K., Federman, S. R., \& Lambert, D. L. 2006, ApJ, 649, 788

Ritchey, A. M., Federman, S. R., \& Lambert, D. L. 2011, ApJ, 728, 36

Scarrott, S. M., Ward-Thompson, D., \& Warren-Smith, R. F. 1987, MNRAS, 224, 299

Schlegel, D. J., Finkbeiner, D. P., \& Davis, M. 1998, ApJ, 500, 525

Serkowski, K., Mathewson, D. L., \& Ford, V. L. 1975, ApJ, 196, 261

Sheffer, Y., Rogers, M., Federman, S. R., et al. 2008, ApJ, 687, 1075

Simon, J. D., Gal-Yam, A., Gnat, O., et al. 2009, ApJ, 702, 1157

Słyk, K., Bondar, A. V., Galazutdinov, G. A., \& Krełowski, J. 2008, MNRAS, 390,1733

Snow, T. P., \& McCall, B. J. 2006, ARA\&A, 44, 367

Sollerman, J., Cox, N., Mattila, S., et al. 2005, A\&A, 429, 559

Sonnentrucker, P., Welty, D. E., Thorburn, J. A., \& York, D. G. 2007, ApJS, 168, 58
Srianand, R., Gupta, N., Rahmani, H., et al. 2013, MNRAS, 428, 2198 Steidel, C. C., Rich, R. M., \& McCarthy, J. K. 1990, AJ, 99, 1476

Sternberg, A., Gal-Yam, A., Simon, J. D., et al. 2011, Science, 333, 856

Stritzinger, M., Burns, C. R., Phillips, M. M., et al. 2010, AJ, 140, 2036

Stritzinger, M. D., Phillips, M. M., Boldt, L. N., et al. 2011, AJ, 142, 156

Thaddeus, P. 1972, ARA\&A, 10, 305

Thorburn, J. A., Hobbs, L. M., McCall, B. J., et al. 2003, ApJ, 584, 339

Vos, D. A. I., Cox, N. L. J., Kaper, L., Spaans, M., \& Ehrenfreund, P. 2011, A\&A, 533, A129

Wang, L., Baade, D., Clocchiatti, A., et al. 2008, Central Bureau Electronic Telegrams, 1509, 1

Wegner, G., Bernardi, M., Willmer, C. N. A., et al. 2003, AJ, 126, 2268

Weiss, R. 1980, ARA\&A, 18, 489

Welty, D. E., \& Crowther, P. A. 2010, MNRAS, 404, 1321

Welty, D. E., Federman, S. R., Gredel, R., Thorburn, J. A., \& Lambert, D. L. 2006, ApJS, 165, 138

Welty, D. E., Howk, J. C., Lehner, N., \& Black, J. H. 2013, MNRAS, 428, 1107

Whittet, D. C. B. 1992, Dust in the galactic environment (IOP Publ.)

Whittet, D. C. B., Martin, P. G., Hough, J. H., et al. 1992, ApJ, 386, 562

York, B. A., Ellison, S. L., Lawton, B., et al. 2006, ApJ, 647, L29 